\title{
Sandpile Model with Activity Inhibition
}

\author{
S. S. Manna ${ }^{1}$ and D. Giri ${ }^{2}$ \\ Department of Physics, Indian Institute of Technology \\ Powai, Mumbai 400076, India
}

(August 13, 2018)

\begin{abstract}
A new sandpile model is studied in which bonds of the system are inhibited for activity after a certain number of transmission of grains. This condition impels an unstable sand column to distribute grains only to those neighbours which have toppled less than $m$ times. In this non-Abelian model grains effectively move faster than the ordinary diffusion (super-diffusion). A novel system size dependent cross-over from Abelian sandpile behaviour to a new critical behaviour is observed for all values of the parameter $m$.
\end{abstract}

05.70.Jk, 05.40.+j, 05.70.Ln

Typeset using REVTEX 
The concept of Self-Organized Criticality (SOC) was introduced to describe how a system, starting from an arbitrary initial condition may evolve to a scale free critical state following some specific dynamical rules while under the action of repeated external perturbations [1]. Naturally occurring physical phenomena like sandpiles [2], forest fires [3], river networks [4], earthquakes [5] etc. are argued as systems showing SOC. To demonstrate the idea of SOC a simple model known as the 'sandpile' model was introduced in which a stochastically driven cellular automata evolves under a non-linear, diffusive, self-organizing mechanism leading to a non-equilibrium critical state [1].

At present many different versions of the sandpile model are available. However precise classification of various models in different universality classes in terms of their critical exponents is not yet fully complete and still attracts much attention [6]. Among the different models most widely studied is the Abelian Sandpile Model (ASM) in which many analytical [7] as well as numerical [8] results are known. Some efforts have also been given towards the analytical calculation of avalanche size exponents $[9,10]$. Secondly a Two-state sandpile model with stochastic evolution rules was also studied [11] which was initially thought to belong to the same universality class as that of ASM $[11,12]$ but later claimed to be different $[13]$.

We consider a situation in which an intermediate time scale is associated with every bond of the system. Each bond allows only a certain number of grains to cross from its one end to the other and after that it has a dead time and cannot support any further traffic untill a new avalanche starts. This dead time is much greater than the time scale of avalanche propagations but much less than the input rate of grains. We call this model as the "Sandpile Model with Activity Inhibition' (SMAI).

Similar to different sandpile models we also define our model on a regular lattice with open boundary. Non-negative integer numbers $\left(h_{i}\right)$ assigned at the lattice sites represent the heights of the sand columns. Sand grains are added at randomly chosen sites by increasing the $h$ values by unity $: h_{i} \rightarrow h_{i}+1$. The possibility of a sand column becoming unstable 
arises only when the height $h_{i}$ becomes greater than a threshold value $h_{c}$. Such a column becomes unstable only if the number $n_{i}$ of nearest neighbour sites which have toppled less than a pre-assigned cut-off number $m$ within the same avalanche is found to be non zero. An unstable column immediately topples and distributes one grain each to all the $n_{i}$ neighbours $: h_{j} \rightarrow h_{j}+1\left(j=1\right.$ to $\left.n_{i}\right)$. The sand column decreases by the same amount $: h_{i} \rightarrow h_{i}-n_{i}$. If $n_{i}=0$, the sand column does not topple and its height though greater than $h_{c}$ is considered stable. In an avalanche sites can topple a maximum of $m$ times. This implies that in the limit of $m \rightarrow \infty$ our model converges to ASM. Recently a Stochastic Sandpile Model (SSM) has been studied in which sand columns having heights greater than the threshold are also considered stable [14].

One unit of time within an avalanche consists of the following intermediate steps : (i) a list of all sites where $h_{i}>h_{c}$ is made (ii) $n_{i}$ values are calculated for each site $i$ (iii) all sites with non-zero $n_{i}$ values are toppled in parallel.

We first consider the case where the cut-off in the toppling number $m=1$. Here the toppling front moves outwards and grains always jump only in the outward direction and do not fall back. Therefore compared to the random walk analogy for the movement of the grains in ASM [15] in our model grains move faster than diffusing particles. This is indeed reflected in the average cluster size $\left\langle s>\sim L^{\beta_{s}}\right.$ where $\beta_{s}=1.62$ (reported below). This implies that the displacements $\mathcal{R}$ of the grains in our model grows with time $\mathcal{T}$ as $\mathcal{R} \sim \mathcal{T}^{\nu}$ with $\nu=1 / 1.62=0.62$ which is faster than diffusion (super-diffusion).

Zhang had studied a scaling theory of the sandpile model in which the toppling front grows as a $(d-1)$ dimensional surface in the $d$ dimension [16]. In this analysis, where multiple topplings were ignored, the exponent for the avalanche size distribution was possible to calculate analytically in all dimensions. Since in SMAI, a single toppling front moves outward and multiple topplings are forbidden for $m=1$, we expect that SMAI may be a correct realization of the Zhang's theory [16].

Unlike ASM our model turns out to be non-Abelian. Different steady state configurations 
are obtained on dropping grains at same locations but following different sequences. We pick up a stable configuration in a $2 \times 2$ cell and drop one grain each at the two opposite corners one after the other (figure 1). On reversing the order of dropping a different stable state configuration is obtained. We notice that non-abelianity is effective only when avalanche cluster sizes are greater than one.

However, it is easy to see that just like in ASM, we cannot have the forbidden subcofigurations (FSC) anywhere in the lattice. An FSC is defined as the subset of connected sites for which at each site the height is less than its coordination number in the subset [7]. In SMAI also two neighbouring sites whose heights are both zero $(0-0)$ will never occur in the steady state because of the same reason as in ASM that if one topples the other site will receive one grain. Similarly a height configuration like $(0-1-0)$ is also an FSC. In fact, all the FSCs defined for ASM are also forbidden here, and a recurrent config must burn completely. This is checked by burning many successive steady state configurations where the fire starts from the boundary as well as from those sites with heights greater than $h_{c}[7]$. All the configurations are observed to burn fully. No unburnt configuration is found which is obtained by adding grains to a steady state that burns.

To use the rotational symmetry of the system the sandpile is grown with $h_{c}=3$ within a circular region of radius $R=(L-1) / 2$ placed on a square lattice of size $L \times L$. In the steady state starting from the boundary the average height grows quickly radially towards the centre following a power law : $\langle h(r)\rangle=A-B(R-r)^{-\delta}$, where $r$ is the radial distance measured from the centre. We estimate $A=2.3904, B=7.81$ and $\delta=0.75$ for $L=1025$ (figure 2). The average height per site is found to depend on $L$ which on plotting with $1 / L$ extrapolates to a value 2.3840 in the limit of $L \rightarrow \infty$. Similar analysis yields the fraction of sites with different column heights are approximately $2.1 \times 10^{-4}(h=0), 0.2421(h=1)$, $0.3059(h=2), 0.3404(h=3)$. Beyond $h=3$, this fraction decreases approximately exponentially as $\exp (-\alpha h)$ where $\alpha=1.64$ and adds up to a total of 0.1118 .

The size of the avalanche is measured in three different ways : (i) the total number sites 
$s$ which crosses the threshold $h_{c}$ (either toppled or not, both counted) (ii) the life time of the avalanche $(t)$ and (iii) the linear extent or the radius $(r)$ of the avalanche. Since $s, t$ and $r$ are the three different measurements of the same random avalanche cluster, they are necessarily dependent variables. These quantities are assumed to depend on one another as

$$
s \sim t^{\gamma_{t s}}, \quad r \sim t^{\gamma t r}, \quad s \sim r^{\gamma_{r s}} .
$$

The three $\gamma$ exponents are connected by the relation

$$
\gamma_{t s}=\gamma_{t r} \gamma_{r s}
$$

To estimate the exponents $\gamma_{t s}$ and $\gamma_{t r}$ we measure the avalanche size $s$ and avalanche radius $r$ at every time step $t$ during the progress of each avalanche. The total number of topplings up to time $t$ gives the intermediate size $s$ where as the size of the smallest square which encloses the cluster gives the intermediate radius $r$. We estimate $\gamma_{t s}=1.64$ and $\gamma_{t r}=0.83$. Since the avalanche clusters are quite compact and has only few small holes it is justified to assume that $\gamma_{r s}=2$. These values very closely satisfy the equation (2).

We assume the finite size scaling forms for the probability distribution functions as

$$
P(s) \sim s^{-\tau_{s}} f_{s}\left(\frac{s}{L^{\sigma_{s}}}\right), \quad P(t) \sim t^{-\tau_{t}} f_{t}\left(\frac{t}{L^{\sigma_{t}}}\right), \quad P(r) \sim r^{-\tau_{r}} f_{r}\left(\frac{r}{L^{\sigma_{r}}}\right) .
$$

Consequently the cumulative probability distribution $F(x)=\int_{x}^{L^{\sigma_{x}}} P(x) d x$ varies as $x^{1-\tau_{x}}$. However, in the case of $\tau_{x}=1$, the variation should be in the form $F(x)=C-\log (x)$.

We plot the data of $F(s)$ in two different ways. In figure 3 we plot $F(s)$ vs. $s$ for system sizes $L=65,257$ and 1025 using a $\log$ - lin scale. Presence of humps in the large $s$ limit is visible for bigger system sizes which reflects the effect of the finite system size on power law distributions. However in the intermediate region curves are reasonably straight indicating that the exponent $\tau_{s}$ is likely to be 1 . We further plot $F(s) s^{\tau_{s}(L)-1}$ with $s$ on a log $-\log$ scale and tune $\tau_{s}(L)$, the effective $\tau_{s}$ exponent for the system size $L$, such that the curves become horizontal in the intermediate range of $s$. All three curves collapse nicely when the abscissa is scaled as $s L^{-1.62}$ which implies that $\sigma_{s}=1.62$. We show in the figure 4 that the 
$\tau_{s}(L)$ values very closely fit to a straight line when plotted with $L^{-1 / 4}$. It seems that $L^{-1 / 4}$ may be the right leading correction to scaling. The fitted straight line when extrapolated to $L \rightarrow \infty$ gives a value of 1.016 for $\tau_{s}$. Similar analysis for the life time distribution also leads us to conclude that $\tau_{t}=1.02, \sigma_{t}=0.98$.

The radius distribution $F(r)$ is calculated in a slightly different way. We estimate the probability that a site at a distance $r$ from the centre of mass of the avalanche cluster is a part of the cluster. We take into consideration the 'degeneracy' effect that different sites could be at exactly equal distances from the centre. In figure 5 we show a scaling plot $F(r) L^{0.20}$ against $r L^{-0.86}$ using a $\log$ - lin scale for different system sizes. Here we see a much better straight part in the intermediate region. We conclude a value of $\tau_{r} \approx 1$.

The distribution functions follow relations like $P(s) d s \sim P(t) d t$ which imply following scaling relations

$$
\tau_{s}-1=\gamma_{t s}\left(\tau_{t}-1\right), \quad \tau_{r}-1=\gamma_{t r}\left(\tau_{t}-1\right), \quad \tau_{s}-1=\gamma_{r s}\left(\tau_{r}-1\right) .
$$

These equations imply that if one of the exponents $\tau_{s}, \tau_{t}$ or $\tau_{r}$ is equal to one, rest are also equal to one, irrespective of the values of the $\gamma$ exponents. Our estimates for the different $\tau$ exponents are very much consistent with these equations. We also observe that the value of $\tau_{s} \approx 1$ agrees very well with the Zhang's result $\tau_{s}=2(1-1 / d)$ for $d=2[16]$.

We also assume that the average values of $s, t$ and $r$ varies with the system size $L$ as

$$
<s(L)>\sim L^{\beta_{s}}, \quad<t(L)>\sim L^{\beta_{t}}, \quad<r(L)>\sim L^{\beta_{r}} .
$$

We plot $<s(L)>$ vs. $L$ on $\log -\log$ scale for $L=33,65,129,257,513$ and 1025. Slopes between successive points are plotted with $L^{-2}$ and extrapolated to $L \rightarrow \infty$ limit giving $\beta_{s}=1.61$. Similar analysis gives $\beta_{t}=0.96$ and $\beta_{r}=0.82$.

Using the scaling forms in equation (3) we get following scaling relations for $\beta$ exponents as

$$
\beta_{s}=\sigma_{s}\left(2-\tau_{s}\right), \quad \beta_{t}=\sigma_{t}\left(2-\tau_{t}\right), \quad \beta_{r}=\sigma_{r}\left(2-\tau_{r}\right) .
$$


With our measured values of $\beta, \sigma$, and $\tau$ these relations are approximately satisfied. We put errors of 0.05 to all our measured exponents.

Next we study the case when cut-off for the toppling number $m>1$. The average cluster size $\langle s(L)>$ is plotted with $L$ on the $\log -\log$ scale in figure 6 for $m=1,2,4$ and 8 . We see that all curves are parallel straight lines with slopes approximately 1.61 for large system sizes. However for small system sizes all of them bend and become part of the same straight line. Then we plot on the same figure the $\langle s(L)>$ data for ASM. We get a straight line with a slope $\approx 2$ which almost overlaps with the bend portions of the curves for different $m$ values. We explain this by noting that for every $m$ value our model behaves as ASM for small system sizes. In small systems the number of avalanches where sites will topple more than $m$ times are very few. However for bigger system sizes the cut-off $m$ will have more prominent effects. Therefore for each $m$ values there should be one particular system size where the cross-over takes place from ASM to Non-abelian behaviour. The cross-over size $L_{c}$ is seen to be roughly proportional to the value of $m$. We expect that for any $m$ value if one works in systems larger than the cross-over size one should get the same set of exponents as those in the case of $m=1$.

To summarize, we studied here a new sandpile model where bonds of the system relax after a certain number of transmission of grains. This limits a site to topple a maximum of $m$ times within the same avalanche. Based on the results of detailed numerical studies using improved algorithms we claim a cross-over from ASM behaviour to a new critical behaviour at a particular size of the system whose magnitude depends on the value of $m$.

We acknowledge with thanks D. Dhar for many useful discussions and suggestions. 


\section{FIGURES}

FIG. 1. Non-abelian property of the sandpile model is shown on a $2 \times 2$ cell. On the same initial stable configuration two grains are added at two different sites but in different orders. Different final stable configurations are obtained.

FIG. 2. The average height profile of the sandpile in a circular region plotted with the distance from the circumference of the circle is shown. The slope of the curve is 0.75 and $A=2.3904$ is found.

FIG. 3. Log - lin plot of the cumulative probability distribution $F(s)$ for the three system sizes $L=65,257$ and 1025 (from left to right). The straight portions of the curves in the intermediate regions indicates that $\tau_{s}$ is likely to be equal to one.

FIG. 4. Plot of $\tau_{s}(L)$ for different system sizes $L=33,65,129,257,513$ and 1025 with $L^{-1 / 4}$. A direct straight line fit gives $\tau_{s}=1.016$ in the $L \rightarrow \infty$ limit.

FIG. 5. Scaling plot of the cumulative radial distribution function $F(r)$. Plot of $F(r) L^{0.20}$ vs. $r / L^{0.86}$ shows the data collapse for the system sizes $L=65,257$ and 1025 .

FIG. 6. Plot of $\langle s(L)>$ versus $L$ for $m=1,2,4$ and 8 of SMAI (solid lines) and for ASM (dot dashed line). For each value of $m$ there is a threshold system size $L$ at which the cross-over from ASM behaviour to SMAI takes place. 


\section{REFERENCES}

${ }^{1}$ Electronic address : manna@niharika.phy.iitb.ernet.in

${ }^{2}$ Electronic address : giri@niharika.phy.iitb.ernet.in

[1] P. Bak, C. Tang and K. Wiesenfeld, Phys. Rev. Lett. 59, 381 (1987); Phys. Rev. A 38, 364 (1988); P. Bak, How Nature Works: The Science of Self-Organized Criticality, (Copernicus, New York, 1996).

[2] G. A. Held, D. H. Solina II, D. T. Keane, W. J. Haag, P. M. Horn and G. Grinstein, Phys. Rev. Lett. 65, 1120 (1990); V. Frette, K. Christensen, A. Malte-Sorensen, J. Feder, T. Josang and P. Meakin, Nature (London) 379, 49 (1996).

[3] P. Bak and K. Chen, Physica D 38, 5 (1989).

[4] H. Takayasu and H. Inaoka, Phys. Rev. Lett. 68, 966 (1992); A. Rinaldo, I. RodriguezIturbe, R. Rigon, E. Ijjasz-Vasquez and R. L. Bras, Phys. Rev. Lett. 70, 822 (1993); S. S. Manna and B. Subramanian, Phys. Rev. Lett. 76 (1996) 3460.

[5] J. M. Carlson and J. S. Langer, Phys. Rev. Lett. 62, 2632 (1989); A. Sornette and D. Sornette, Europhys. Lett. 9, 197 (1989); Z. Olami, H. J. S. Feder and K. Christensen, Phys. Rev. Lett. 68, 1244 (1992).

[6] L. P. Kadanoff, S. R. Nagel, L. Wu and S. Zhou, Phys. Rev. A. 39, 6524 (1989); S. S. Manna Physica A 179, 249 (1991).

[7] D. Dhar, Phys. Rev. Lett. 64, 1613 (1990); S. N. Majumdar and D. Dhar 185, 129 (1992); E. V. Ivashkevich, D. V. Ktitarev and V. B. Priezzhev, Physica A 209, 347 (1994).

[8] S. S. Manna, J. Stat. Phys., 59, 509 (1990); P. Grassberger and S. S. Manna, J. Phys. (France) 51, 1077 (1990). 
[9] V. B. Priezzhev, D. V. Ktitarev, and E. V. Ivashkevitch, Phys. Rev. Lett. 76, 2093 (1996).

[10] ] M. Paczuski and S. Boettcher, cond-mat/9705174.

[11] S. S. Manna, J. Phys. A 24, L363 (1992).

[12] A. Vespignani, S. Zapperi and L. Pietronero, Phys. Rev. Lett. 72, 1690 (1994); Phys. Rev. E 51, 1711 (1995).

[13] A. Ben-Hur and O. Biham, Phys. Rev. E. 53, R1317 (1996); H. Nakanishi and K. Sneppen, Phys. Rev. E. 55, 4012 (1997).

[14] B. Tadic and D. Dhar, TIFR/TH/97-17 preprint.

[15] D. Dhar, Physica A 186, 82 (1992).

[16] Y-C. Zhang, Phys. Rev. Lett. 63, 470 (1989). 
(a)

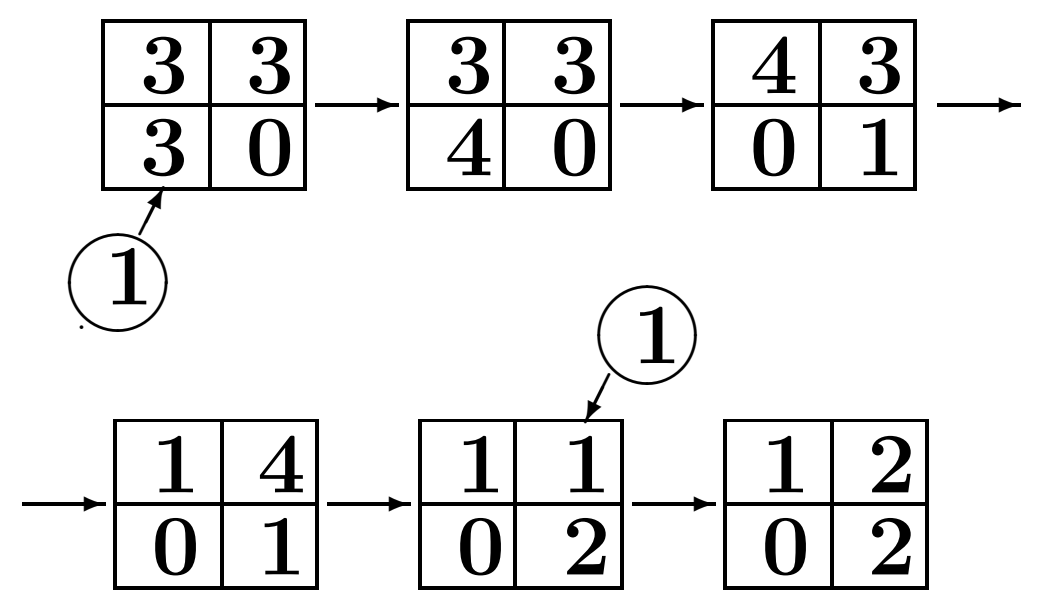

(b)

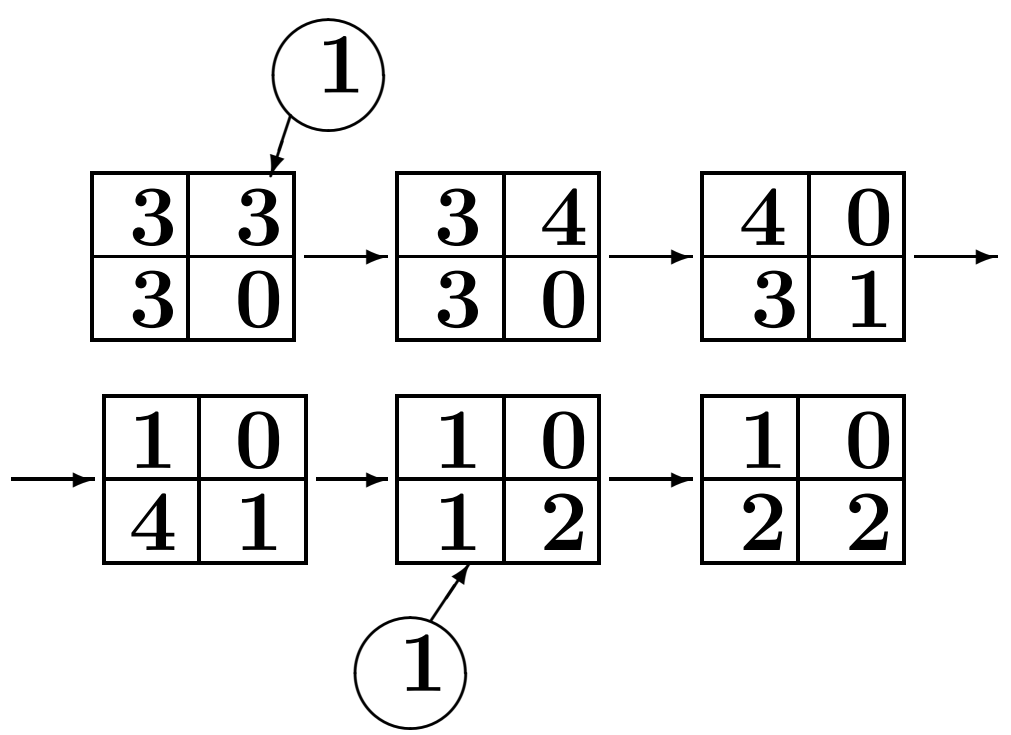

Figure 1: Manna and Giri 


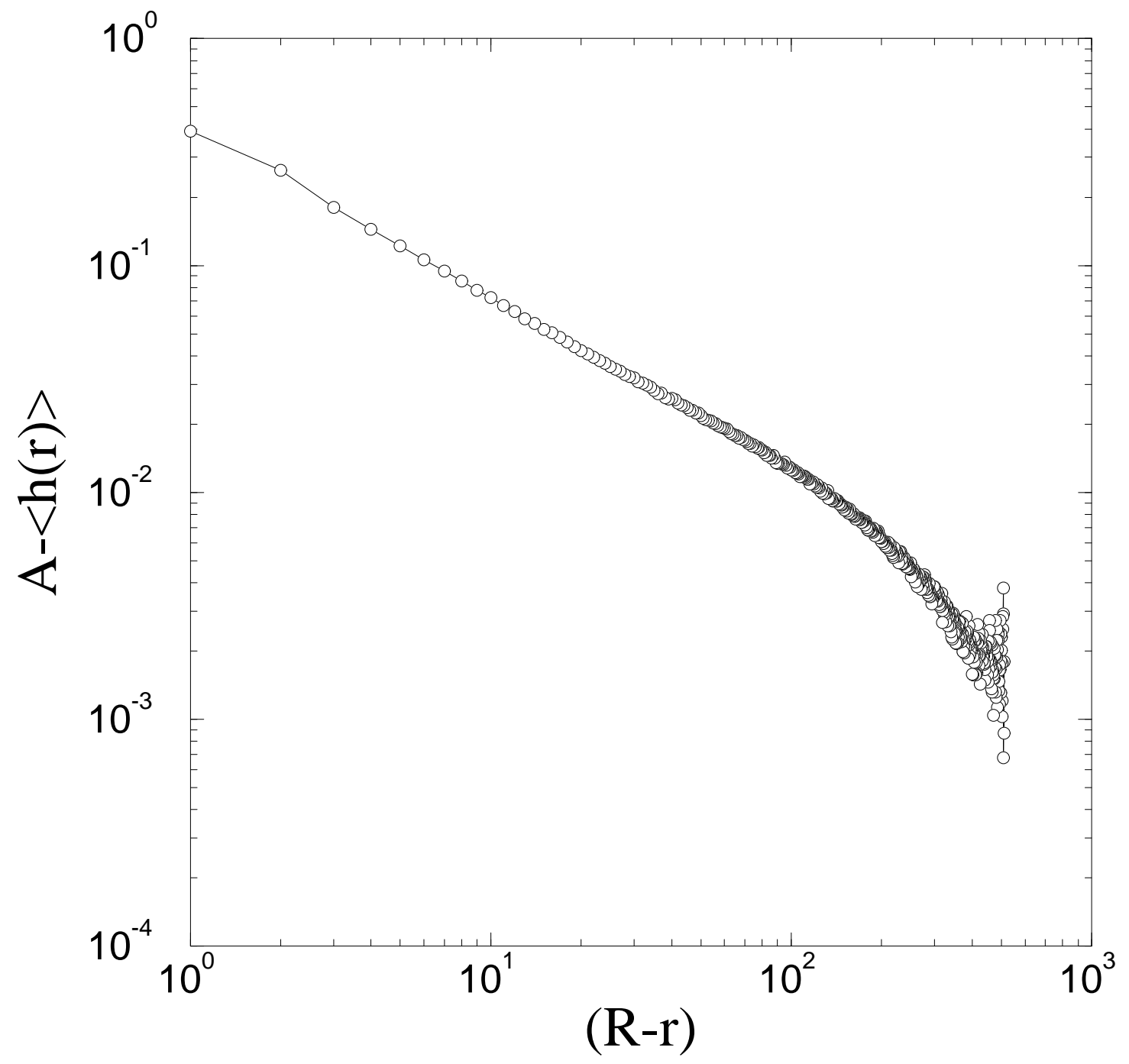

Figure 2 : Manna and Giri 


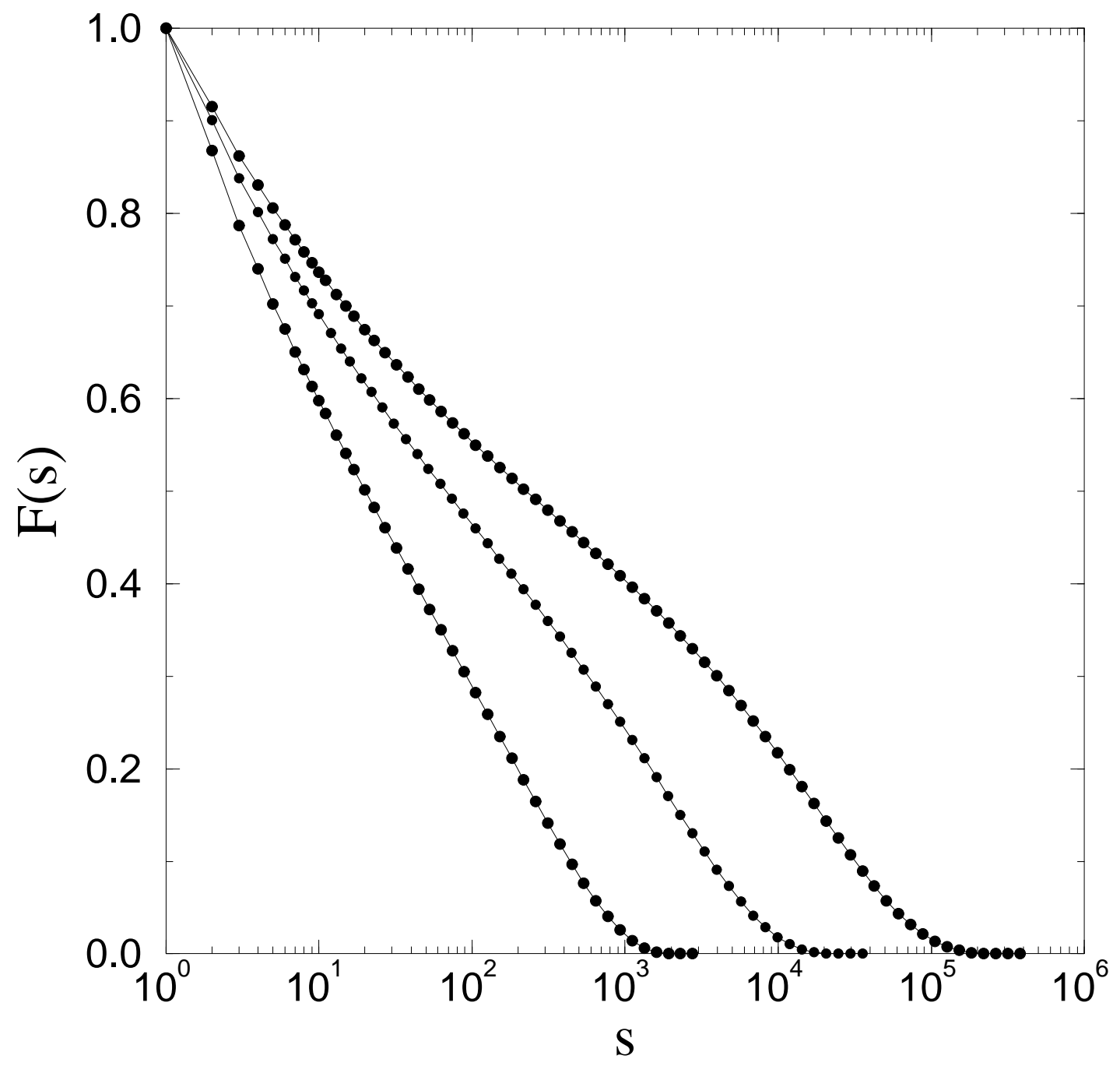

Figure 3 : Manna and Giri 


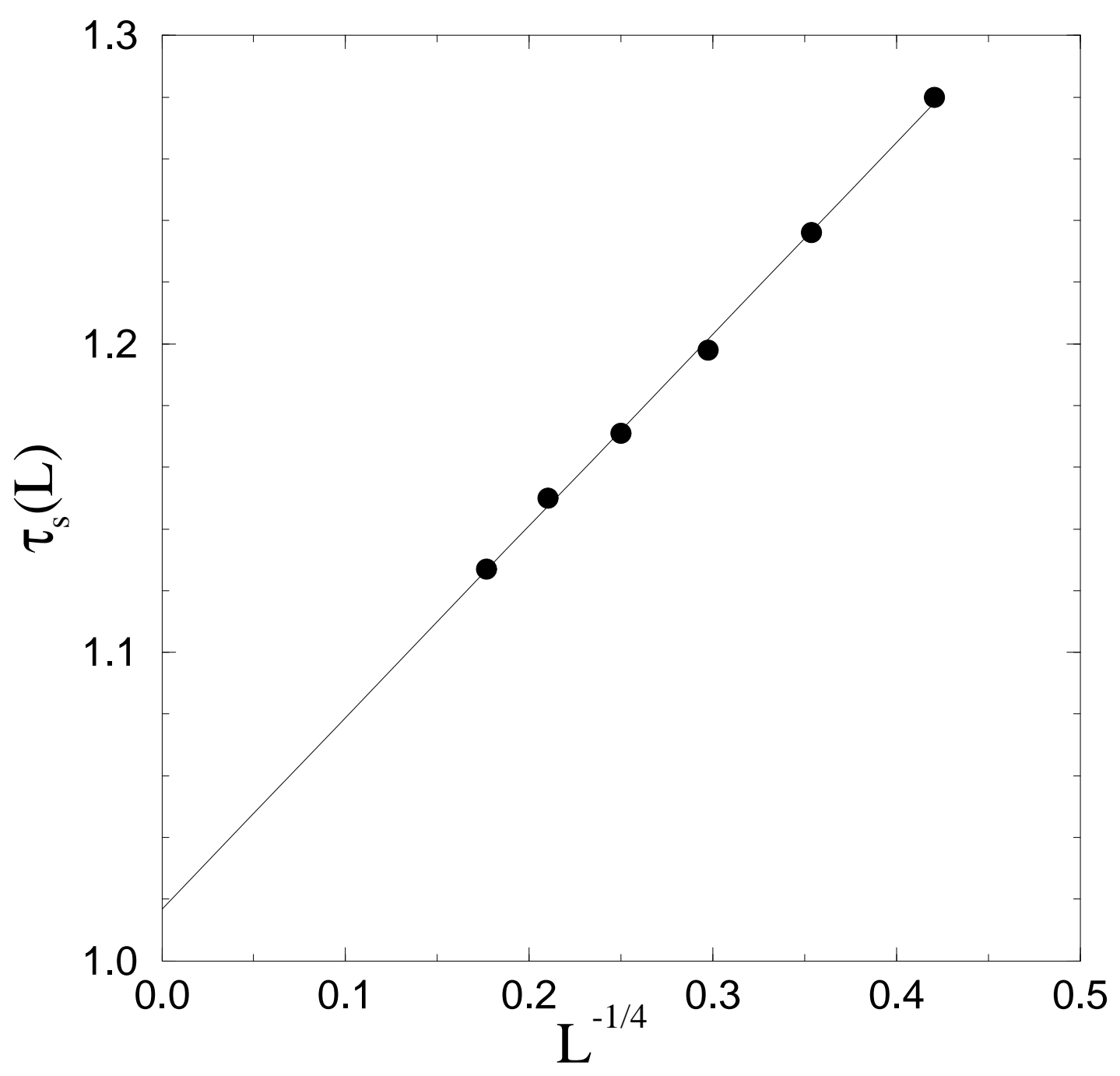

Figure 4 : Manna and Giri 


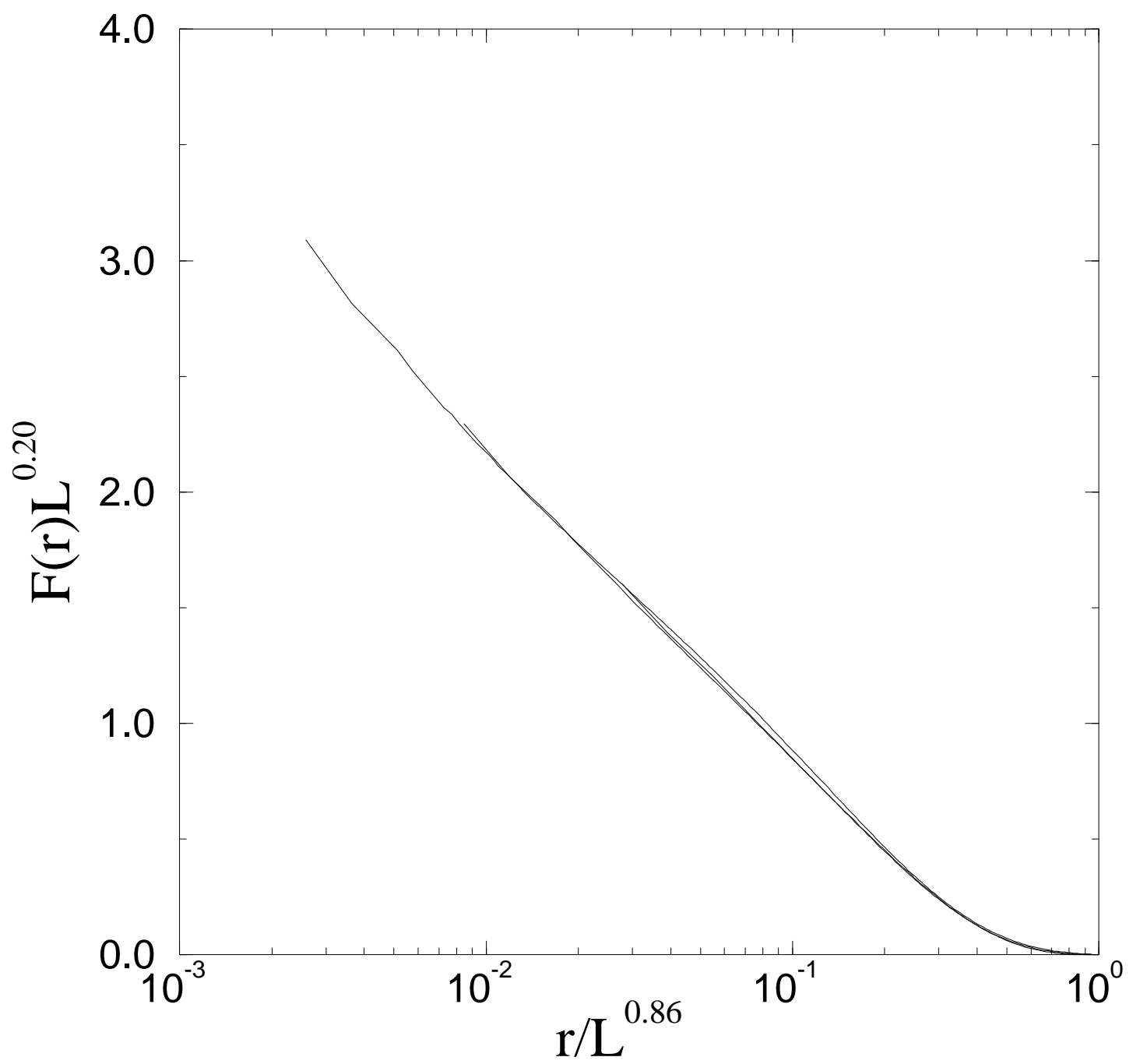

Figure 5 : Manna and Giri 


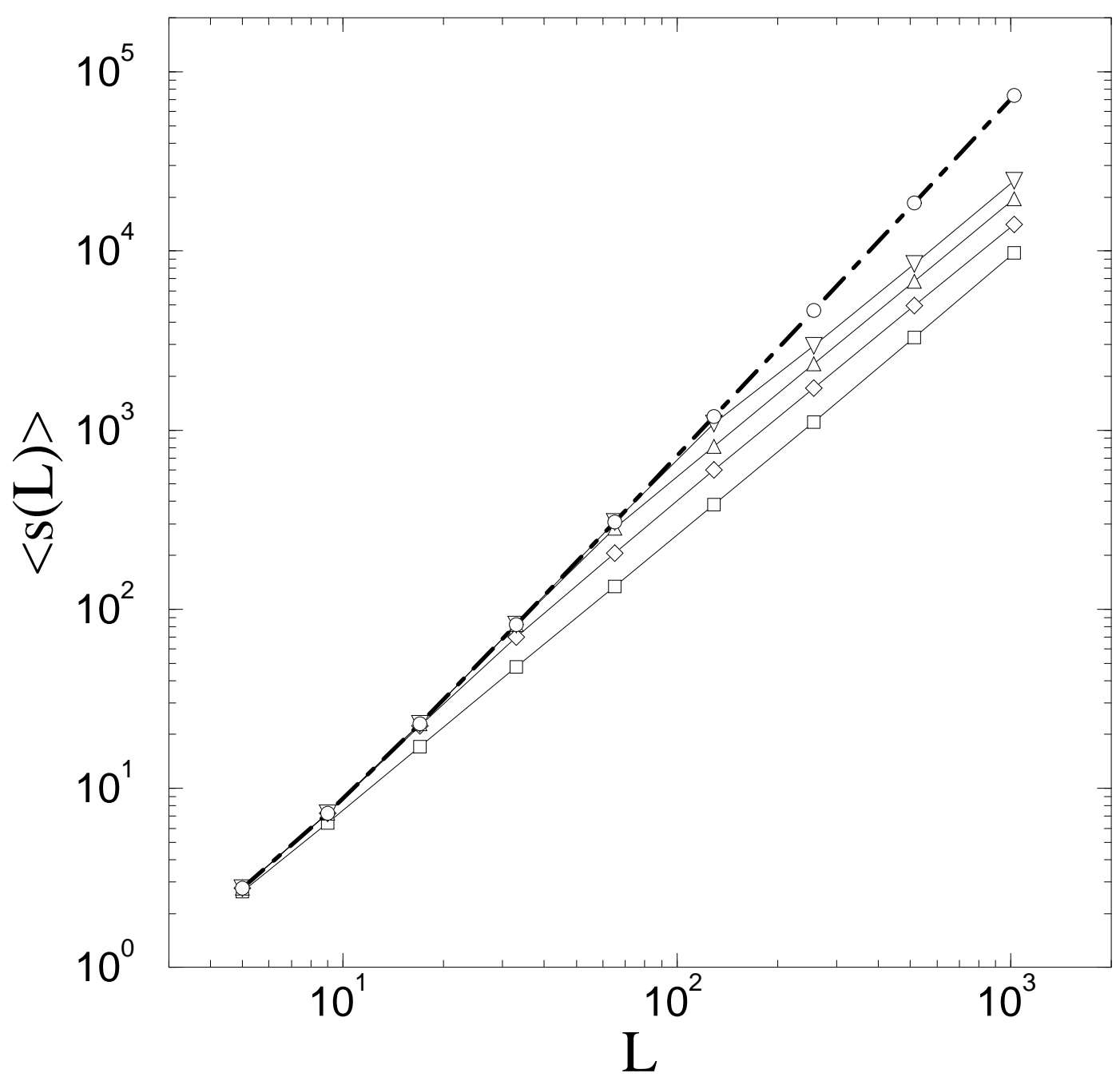

Figure 6 : Manna and Giri 